\title{
The Ventilator Discontinuation Process: An Expanding Evidence Base
}

\author{
Neil R MacIntyre MD FAARC
}

\author{
Introduction \\ Why Might It Be Difficult to Withdraw Patients From the Ventilator? \\ Scope of the Problem \\ The Evidence Base Supporting Ventilator Withdrawal Strategies in the \\ Intensive Care Setting \\ The Evidence Base Supporting Ventilator Withdrawal Strategies in the \\ PMV Patient \\ Can the Ventilator Withdrawal Process Be Automated? \\ Assessing the Need for an Artificial Airway \\ The Role of Tracheostomies \\ Summary
}

\begin{abstract}
The ventilator discontinuation process is an essential component of overall ventilator management. Undue delay leads to excess stay, iatrogenic lung injury, unnecessary sedation, and even higher mortality. On the other hand, premature withdrawal can lead to muscle fatigue, dangerous gas exchange impairment, loss of airway protection, and also a higher mortality. Continued ventilator dependence can be a result of persistent illness or can be a result of poor management. It is obviously important for the clinician to be able to assess both of these issues. An evidence-based task force has recommended regular assessments focusing on the causes of ventilator dependence, regular assessments for evidence of disease stability/reversal, use of regular spontaneous breathing trials (SBTs) as the primary assessment tool for ventilator discontinuation potential, use of separate assessments to evaluate the need for an artificial airway in patients tolerating the SBT, and the use of comfortable, interactive ventilator modes (that do not need to be "weaned") in between regular SBTs. More recent developments have focused on the importance of linking sedation reduction protocols to ventilator discontinuation protocols. Patients with repeated SBT failures are often considered to require prolonged mechanical ventilation (PMV). These patients often receive tracheostomies and are probably better managed with more gradual reductions in support and gradually lengthened spontaneous breathing periods. PMV patients have a high 1-year mortality, and many may ultimately require lifelong support. This evidence base is growing, but the earlier guidelines are standing the test of time. Indeed, practice patterns are evolving in accordance with them. Nevertheless, there is still room for improvement, and further clinical studies, especially in the patient requiring PMV, are needed. Key words: prolonged mechanical ventilation; discontinuation; ventilator management; iatrogenic lung injury; sedation; ventilator dependence; spontaneous breathing trial. [Respir Care 2013;58(6):1074-1082. (C) 2013 Daedalus Enterprises]
\end{abstract}

Dr MacIntyre is affiliated with the Division of Pulmonary and Critical Care Medicine, Duke University Hospital, Durham, North Carolina.

Dr MacIntyre presented a version of this paper at the 51st RESPIRATORY CARE Journal Conference, "Adult Mechanical Ventilation in Acute Care: Issues and Controversies," held September 7 and 8, 2012, in St Petersburg, Florida.
Dr MacIntyre has disclosed a relationship with CareFusion.

Correspondence: Neil R MacIntyre MD FAARC, Division of Pulmonary and Critical Care Medicine, Duke University Hospital, Box 3911, Durham NC 27710. E-mail: neil.macintyre@duke.edu.

DOI: $10.4187 /$ respcare. 02284 


\section{Introduction}

Patients require positive pressure mechanical ventilator support through an artificial airway for many reasons. Regardless of the reason, however, as the situation stabilizes and begins to reverse, clinical attention should shift to the ventilator withdrawal process. Failure to recognize ventilator withdrawal potential will result in longer stay, higher costs, excessive sedation, longer exposure to potentially "toxic" airway pressures/volumes, and increased infection risk. ${ }^{1-4}$ On the other hand, overly aggressive ventilator withdrawal attempts carry their own hazards. Specifically, premature ventilator withdrawal can lead to airway loss, compromised gas exchange, aspiration, and inspiratory muscle fatigue. ${ }^{5,6}$ Indeed, a failed extubation is associated with an 8-fold higher odds ratio for nosocomial pneumonia and a 6-12-fold increased mortality risk. ${ }^{7,8}$

The clinical challenge then is to balance aggressiveness with safety. A common quality indicator addressing this balance is the reintubation rate (ie, patients needing reintubation/total number of patients extubated). A value too low suggests unnecessary delays in ventilator removal; a value too high suggests inappropriate aggressiveness in support removal. Reported reintubation rates range from $4 \%$ to $23 \%$ for different ICU populations, and may be as high as $33 \%$ in patients with mental status changes and neurologic impairment. ${ }^{3,8-15}$ Although never subjected to rigorous cost/benefit analyses, reintubation rates of 5-20\% are generally considered reasonable.

This paper will review several concepts involved in the ventilator withdrawal process. These include the causes of ventilator dependence, the scope of the problem, and the evidence supporting current recommendations for ventilator withdrawal strategies across a variety of clinical settings.

\section{Why Might It Be Difficult to Withdraw Patients From the Ventilator?}

In concept, there are 2 fundamental reasons that patients are unable to be withdrawn from mechanical ventilator support. ${ }^{16}$ The first is that they are "too sick." This is illustrated in Figure 1, ${ }^{17}$ which defines ventilator dependence as being caused by an imbalance between demands and capabilities. High demands come from high ventilation/oxygenation requirements and/or abnormal respiratory system mechanics. Impaired capabilities come from poor neural ventilatory control and/or compromised respiratory muscle function. Depending upon the disease state and underlying health status, this imbalance can resolve quickly (eg, drug overdose) or can require weeks to months to recover. Indeed, in some patients the imbalance is never restored and life-long mechanical ventilation is required.

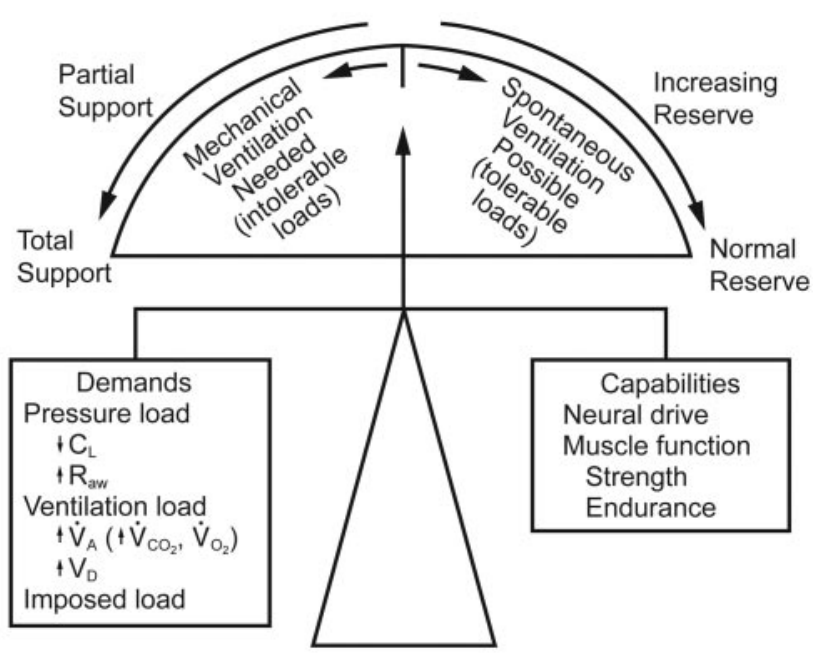

Fig. 1. Schematic representation of the balance between patient demands and capabilities. When demands outweigh capabilities, mechanical ventilation is needed. When capabilities outweigh demands, ventilator discontinuation is possible. (From Reference 17).

The second fundamental reason that patients are unable to be withdrawn is clinician behavior. ${ }^{16}$ Large clinical trials from the 1990s clearly showed that clinicians frequently did not appreciate in a timely fashion that ventilator withdrawal was possible. This was often made worse by slow "weaning" procedures that actual delayed the withdrawal process. ${ }^{13,14}$ Clinicians can also adversely affect the demand/capability balance in Figure 1 by producing ventilator-induced lung injury from non-lung-protective settings, ${ }^{18}$ by increasing imposed loads from asynchronous ventilator settings, ${ }^{19}$ or by inappropriately blunting neural drive (eg, sedatives/paralytics) ${ }^{20}$ or weakening muscles (eg, toxins, poor nutrition).

\section{Scope of the Problem}

A large observational study from Europe involved 4,559 mechanically ventilated patients from 349 ICUs in 2004. ${ }^{21}$ These data were analyzed using a newly described classification system of ventilator discontinuation22: "Simple" (ventilator discontinued after the first assessment), "Difficult" (ventilator discontinued from 2-7 d after initial assessment), and "Prolonged" (ventilator discontinued in $>7 \mathrm{~d}$ after initial assessment). In this analysis, 2,714 patients were successfully discontinued over the 28 day study period. ${ }^{23}$ Fifty-five percent were simple discontinuation, 39\% were difficult discontinuation, and $6 \%$ were prolonged discontinuation. Not surprisingly, patients needing prolonged discontinuation processes were sicker and had longer stay and higher mortality than the simple discontinuation patients. 
Patients requiring mechanical ventilation beyond the 28 day mark are often considered to be requiring prolonged mechanical ventilation (PMV). The PMV population is growing rapidly and reflects a number of factors, including the aging of the general population and the ability of the healthcare system to keep patients alive longer after devastating illnesses or aggressive surgical procedures. ${ }^{24-26}$ PMV patients are estimated to be as many as $5-10 \%$ of all mechanically ventilated patients in the United States. While in-patient mortality in PMV patients may be as high as $35 \%$, as many as half of the survivors will be successfully withdrawn from mechanical ventilator support, usually within the first 90 days. ${ }^{24}$ Thereafter, the likelihood of successful ventilator withdrawal is very low.

\section{The Evidence Base Supporting Ventilator Withdrawal Strategies in the Intensive Care Setting}

The important clinical questions facing the clinician in the ICU are: When can efforts to discontinue ventilation be initiated? What assessment strategies will best identify the patient who is ready for ventilator discontinuation? When should extubation be carried out?

Evidence to answer these questions comes largely from observational studies in which a certain parameter (or set of parameters) is compared in a group of patients who either successfully or unsuccessfully have been removed from the ventilator. The general goal of these studies is to find "predictors" of outcome. Evaluating results from these types of studies can be difficult for several reasons. ${ }^{27}$ First, the "aggressiveness" of the clinician/investigator's discontinuation philosophy needs to be understood, as it will affect the performance of a given predictor.

Second, patients are recruited into these studies because investigators believe there is some reasonable chance of success for ventilator discontinuation. These "entry" criteria often include some form of clinical judgment or intuition, making results from one study difficult to compare to another.

Third, methodological problems inherent to observational studies include different measurement techniques of a given parameter from study to study, large coefficients of variation of a given parameter with repeated measurements from study to study, and different patient populations (eg, long-term vs short-term ventilator dependence).

Fourth, assessed outcomes differ from study to study. Some investigators have examined successful tolerance of a spontaneous breathing trial (SBT), others have used permanent discontinuation of the ventilator, and others have combined successful discontinuation and extubation. In addition, different studies use different durations of ventilator discontinuation or extubation to define success or failure. Although $24-48 \mathrm{~h}$ of unassisted breathing often is considered to define the successful discontinuation of ven- tilator support in the ICU setting, many studies use shorter time periods to indicate success and often do not report subsequent reintubation rates or the need to reinstitute mechanical ventilatory support.

In 1999, McMaster University, funded by a large grant from the United States Agency for Health Care Policy Research, published a comprehensive evidence-based review of the world literature pertaining to ventilator discontinuation (over 5,000 publications). ${ }^{28}$ This report found evidence in the literature supporting a possible role for 66 specific measurements as predictors of successful ventilator discontinuation. To evaluate the role of these parameters, the McMaster report used likelihood ratios (LRs), an expression of the odds that a given test result will be present in a patient with a given condition, compared to a patient without the condition. An LR $>1$ indicates that the probability of success increases, while a value $<1$ indicates that the probability of failure increases. LRs between 0.5 and 2 indicate that a discontinuation parameter is associated with only small, clinically unimportant changes in the post-test probability of success or failure. In contrast, LRs of $>10$ or $<0.1$ correlate with very large changes in probability. With this approach, the McMaster group identified 7 parameters that had consistently significant LRs to predict successful ventilator discontinuation in several studies. Some of these measurements are made while the patient is still receiving ventilatory support; others require an assessment during a brief period of spontaneous breathing. These parameters, their threshold values, and the range of reported LRs are given in the Table. It should be noted that, despite the statistical significance of these parameters, the generally low LRs indicate that the clinical applicability of these parameters alone to individual patients is low.

Following the publication of the McMaster report, the American College of Chest Physicians, the Society of Critical Care Medicine, and the American Association for Respiratory Care (ACCP/SCCM/AARC) assembled a task force to issue evidence-based guidelines for clinicians to follow in the ventilator discontinuation process. ${ }^{1}$ These guidelines were based largely on the McMaster review of the clinical evidence base, but by necessity also incorporated evidence from basic science work, lung model studies, animal studies, non-outcome-based human studies, and even expert opinion to "fill in the gaps" in the clinical evidence base. In the end, 12 guidelines were described and published. ${ }^{1}$

According to these guidelines, the first step in the discontinuation process is to regularly assess the status and trajectory of underlying cause(s) for the need for mechanical ventilatory support in a given patient. ${ }^{1}$ Among the more important factors impacting ventilator dependence are neurologic abnormalities affecting the brainstem ventilatory control system, respiratory muscle capability/me- 
The Ventilator Discontinuation Process: An Expanding Evidence Base

Table. Parameters in the McMaster Review With Significant Likelihood Ratios for Predicting Ventilator Discontinuation Success

\begin{tabular}{|c|c|c|c|c|}
\hline Parameter & Measurement Method & $\begin{array}{l}\text { Number } \\
\text { of Studies }\end{array}$ & Threshold & $\begin{array}{l}\text { Likelihood } \\
\text { Ratio Range* }\end{array}$ \\
\hline Minute volume & Measured on ventilator & 20 & $10-15 \mathrm{~L} / \mathrm{min}$ & $0.8-2.37$ \\
\hline $\begin{array}{l}\text { Negative inspiratory force (maximum } \\
\text { inspiratory pressure) }\end{array}$ & Measured on ventilator & 26 & -20 to $-30 \mathrm{~cm} \mathrm{H}_{2} \mathrm{O}$ & $0.23-3.01$ \\
\hline $\mathrm{P}_{0.1} /$ maximum inspiratory pressure & Measured on ventilator & 4 & 0.30 & $2.14-25.3$ \\
\hline $\begin{array}{l}\text { CROP (compliance, rate, oxygenation, and } \\
\text { pressure) index }\end{array}$ & Measured on ventilator & 2 & 13 & $1.05-19.74$ \\
\hline Breathing frequency & $\begin{array}{l}\text { Measured during } 1-2 \text { min spontaneous } \\
\text { breathing trial }\end{array}$ & 24 & 30-38 breaths/min & $1.0-3.89$ \\
\hline Tidal volume & $\begin{array}{l}\text { Measured during } 1-2 \text { min spontaneous } \\
\text { breathing trial }\end{array}$ & 18 & $325-408 \mathrm{~mL}(4-6 \mathrm{~mL} / \mathrm{kg})$ & $0.71-3.83$ \\
\hline Breathing frequency/tidal volume & $\begin{array}{l}\text { Measured during } 1-2 \text { min spontaneous } \\
\text { breathing trial }\end{array}$ & 20 & $60-105$ & $0.84-4.63$ \\
\hline
\end{tabular}

chanical load imbalances, impaired ventilation/perfusion matching in the lungs, abnormal cardiac function, lung edema, metabolic derangements (eg, glucose homeostasis and adrenal function), ultimate oxygen delivery, and even psychological factors. A focused search for the underlying causes for ventilator dependence may be especially important in "difficult to discontinue" patients, as previously unrecognized but reversible conditions may be discovered. ${ }^{29}$ As noted above, iatrogenic factors such as excessive sedation use, inappropriate ventilator settings causing lung injury and/or discomfort, inappropriate fluid management, inadequate nutrition, and lack of patient physical activity may also contribute to ventilator dependence. And, of course, a failure to recognize ventilator discontinuation potential will also lead to iatrogenic ventilator dependence.

The criteria used by clinicians to define disease "reversal," however, have been neither defined nor prospectively evaluated in a randomized controlled trial. Rather, various combinations of subjective assessment and objective criteria (eg, usually gas exchange improvement, mental status improvement, neuromuscular function assessments, and radiographic signs) that may serve as surrogate markers of recovery have been employed.3,9-11,13,14,30 It should be noted, however, that some patients who have never met one or more of these criteria still have been shown to be capable of eventual liberation from the ventilator. ${ }^{11}$

Although there needs to be some evidence of "clinical" stability/reversal, a more focused assessment is needed before deciding to continue or discontinue ventilatory support. The ACCP/SCCM/AARC guidelines ${ }^{1}$ state that this formal assessment should be an SBT. This is based on the very strong evidence that, although assessments that are performed while a patient is receiving substantial ventila- tory support or during a brief period of spontaneous breathing can yield important information about discontinuation potential (LRs in the Table), integrated assessments that are performed during a formal, carefully monitored SBT appear to provide the most useful information to guide clinical decision making regarding discontinuation. ${ }^{1}$ In concept, the SBT should be expected to perform well, as it is the most direct way to assess a patient's performance without ventilatory support. Multiple studies have found that patients tolerant of SBTs were found to have successful discontinuations at least $77 \%$ of the time. ${ }^{1,3,9,13,15,29,31}$ However, because patients failing the SBTs in these studies were not systematically removed from ventilatory support, the ability of a failed SBT to predict the need for ventilator dependence (ie, negative predictive value) cannot be formally assessed. Indeed, it is conceivable that iatrogenic factors such as endotracheal tube discomfort or demandvalve insensitivity/unresponsiveness, rather than true ventilator dependence, caused the failure of the SBT in at least some of these patients. ${ }^{3,15,32-35}$ Thus, it is unclear how many patients who are unable to tolerate an SBT would still be able to tolerate long-term ventilator discontinuation. Although the number is likely to be small, it is probably not zero, and this needs to be considered when dealing with patients who repeatedly fail an SBT.

The criteria used to define SBT "tolerance" should be integrated indexes, since, as noted above, single parameters alone perform so poorly. These integrated indexes usually include several physiologic parameters (eg, gas exchange, ventilator pattern, hemodynamics) as well as clinical judgment incorporating such difficult-to-quantify factors as "anxiety," "discomfort," and "clinical appearance." Importantly, these parameters need to be assessed 
in the context of each other and as changes from baseline, not as rigid thresholds. Indeed, one important recent study addressing this issue showed clearly that the use of a rigid threshold of the ratio of breathing frequency to tidal volume $<105$ to define SBT success in fact slowed the discontinuation process. ${ }^{33}$ This observation has implications both for written management protocols as well as computer driven protocols.

The evidence is strong supporting the recommendation that an SBT should be at least 30 min but no longer than $120 \mathrm{~min}$ to allow proper assessment of ventilator discontinuation potential. ${ }^{1}$ This means that clinicians should wait at least $30 \mathrm{~min}$ to assure SBT tolerance, but terminate the trial at $120 \mathrm{~min}$ if SBT tolerance is still unclear. There is evidence that the detrimental effects of ventilator muscle overload, if they occur, often occur early in the SBT. ${ }^{15,34-36}$ Thus, the initial few minutes of an SBT should be monitored closely before a decision is made to continue (this is sometimes referred to as the "screening" phase of an SBT).

Controversy exists on the "best" technique used to do the SBT. Options include a simple T-piece, where only supplemental $\mathrm{O}_{2}$ is supplied at the proximal end of the endotracheal tube, setting the ventilator to a CPAP level equivalent to the previous PEEP setting, or setting a low level of assistance (eg, pressure support of 5-8 $\mathrm{cm} \mathrm{H}_{2} \mathrm{O}$ or the use of "automatic" tube or airway compensation). The T-piece approach comes closest to mimicking the situation the patient will experience when extubated, and it is recommended by some for maximizing specificity of the test (ie, lowest number of false positives). However, because the endotracheal tube is still present with its associated discomfort, the sensitivity of the SBT (ie, detecting true positives) may be somewhat compromised. In contrast, supplying low levels of inspiratory and/or expiratory pressure may hide a patient's inability to tolerate complete ventilator removal (excessive false positive tests), although it may relieve some of the iatrogenic discomfort of the endotracheal tube (fewer false negative tests). In large population studies, all 3 approaches appeared to perform well, but the T-piece approach might be considered if there is concern about borderline SBT performance with other techniques or there is concern about the potential effects from a loss of PEEP. . $^{9} 36-40$

A potential concern about the SBT is safety. Although unnecessary prolongation of a failing SBT conceivably could precipitate muscle fatigue, hemodynamic instability, discomfort, or worsened gas exchange, ${ }^{41-45}$ there are really no data showing that SBTs contribute to any adverse outcomes if terminated promptly when failure is recognized. Indeed, in a cohort of $>1,000$ patients in whom SBTs were routinely administered and properly monitored as part of a protocol, only one adverse event was thought to be even possibly associated with the SBT. ${ }^{11}$
While ICU patients who are judged tolerant of the SBT should move on to assessments of the need for continued use of the artificial airway (see below), the patient who is judged not tolerant of the SBT requires a different approach. In these patients there are 3 specific issues to address. ${ }^{1}$ First, a careful search once again should be undertaken for ongoing (and potentially reversible) causes of ventilatory dependence. Second, a comfortable interactive form of ventilatory support should be provided that encourages respiratory muscle activity but does not overload muscles or compromise gas exchange. ${ }^{19}$ Importantly, there are few (if any) data demonstrating that attempts to "wean" this support, rather than keep it constant, are beneficial. ${ }^{16}$ Third, every 24 hours the patient should be reassessed for another SBT. Importantly, all of these procedures can be carried out through protocols run by skilled clinicians (eg, respiratory therapists). ${ }^{1,11,30}$ Indeed, protocols have been shown in multiple studies to facilitate the ventilator withdrawal process and shorten the duration of mechanical ventilation. ${ }^{46,47}$

Since the publication of the original ACCP/SCCM/ AARC guidelines, ${ }^{1} 2$ important developments have occurred that build on the SBT approach. The first has been the application of computer driven assessments and clinician feedback tools that adjust support and remind clinicians when SBTs are needed..$^{48,49}$ These tools are discussed in more detail below, but their most important feature may be simply to remind clinicians to perform regular SBTs. ${ }^{16}$ Second, and perhaps more importantly, has been the linkage of the SBT strategy to a sedation optimization strategy. ${ }^{50,51}$ As noted above, excessive sedation use has been recognized for years to be a barrier to effective SBT performance and efficient ventilator withdrawal. Optimizing patient-ventilator synchrony with appropriate ventilator settings can help minimize sedation use, but several recent studies have also emphasized that focused protocols aimed at reducing sedation usage further can add to this. Examples of such protocols link the SBT to either spontaneous awakening trials (the routine cessation of all sedatives) ${ }^{50}$ or to focused protocols aimed at minimizing sedation. ${ }^{51}$ An example of a combined SBT and spontaneous awakening trial protocol is given in Figure 2. Regardless of the sedation minimization strategy used, this linkage to routine SBTs has been shown to markedly accelerate the ventilator withdrawal process..$^{50,51}$

It has been over a decade since this comprehensive set of evidence-based guidelines for the ventilator discontinuation process was issued. ${ }^{1}$ The most interesting assessments of the early impact of these guidelines were 2 reanalyses of a large observational database noted above, which involved 4,559 mechanically ventilated patients from 349 ICUs in $2004 .{ }^{21}$ In the first re-analysis ${ }^{52}$ the use of SBTs as the first discontinuation assessment technique was found to have increased from $58 \%$ to $62 \%$ from a similar 


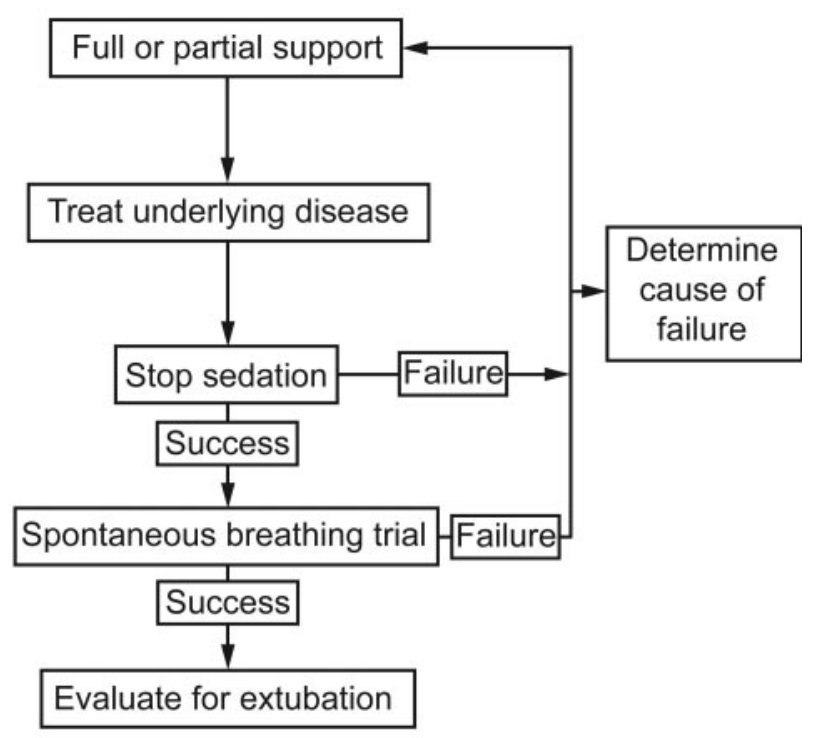

Fig. 2. A conceptual "backbone" to a combined spontaneous awakening trial and spontaneous breathing trial ventilator discontinuation protocol. (From Reference 16.)

survey in $1998(P=.09)$. In the second re-analysis, ${ }^{52}$ discontinuation strategies using gradual support reductions (synchronized intermittent mandatory ventilation [SIMV] or SIMV plus pressure support) were reduced significantly, from $11 \%$ and $26 \%$, respectively, in 1998 , to $1.6 \%$ and $15 \%$, respectively, in $2004(P<.001)$. Importantly, time devoted to the discontinuation process decreased from $50 \%$ to $40 \%$ of total ventilation time over the same period $(P<.001)$

Taken together, these data suggest that clinical use of SBTs is commonplace and gradually increasing, especially in patients judged to be clinically ready for discontinuation. However, there still appears to be a persistent aversion to SBTs in the majority of patients about whom clinicians have concerns. This may not be optimal and, indeed, the evidence would suggest that patients should not be labeled difficult or prolonged discontinuation problems until they have failed multiple SBTs.

\section{The Evidence Base Supporting Ventilator Withdrawal Strategies in the PMV Patient}

The PMV patient is generally a patient who has already failed multiple SBTs and thereby has declared him/herself to have a very slowly resolving disease process. ${ }^{24-26}$ Under these conditions, repeated daily SBTs are likely futile until substantial disease resolution has occurred and the balance in Figure 1 is clearly improving. In the PMV patient, gradual ventilator support reduction strategies ("weaning") now may make some sense. Importantly, these support reduc- tion strategies are not therapeutic: they are not designed to improve the load/capabilities balance of Figure 1 in and of themselves. Instead, tolerance of support reduction strategies would be used to assess disease resolution to determine when it is appropriate to resume SBTs. Although no clinical trials exist in this arena, most authorities would recommend that tolerance of a reduction of support to $50 \%$ of the original level is a signal to restart SBTs. ${ }^{24}$

Because most PMV patients have tracheostomies in place, the SBT strategy is often different from the acute care setting. Indeed, it is usually not a 30-120 min formal trial, but, rather, a gradually increasing duration of tracheostomy collar breathing. The initial trials are often short and are subsequently lengthened until the patient tolerates being off the ventilator during waking hours. Thereafter, attempts at unassisted tracheostomy collar breathing through the night can be attempted.

\section{Can the Ventilator Withdrawal Process Be Automated?}

In recent years a number of feedback systems have been introduced that are designed to automatically reduce the level of ventilator support in patients recovering from respiratory failure..$^{15,48,49}$ These modes usually provide pressure support breaths, and the applied pressure is reduced automatically. Different algorithms are available, and include simple tidal volume targets, tidal volume targets with rate and inspiratory/expiratory time considerations, and tidal volume targets with rate and end-tidal $\mathrm{CO}_{2}$ considerations.

Importantly, with all of these support reduction strategies the assumption is that support reduction is useful before performing SBTs-an assumption that has little supporting evidence in the ICU. ${ }^{16}$ Nevertheless, clinical evidence exists that these modes function as designed and that in settings with rapidly recovering patients and low clinical staffing they may help alert clinicians that SBTs are indicated. Good evidence showing better outcomes with these approaches, compared to proper evidence-based withdrawal strategies in more general ICU settings, does not exist.

Clinicians using these automated support reduction strategies need to understand them well. This is particularly true if using systems based only on a tidal volume target. With this approach, an anxious or uncomfortable patient may generate enough tidal volume that the needed pressure support is actually reduced inappropriately. ${ }^{53}$ If these automated systems have any utility, it would most likely be either in the setting of a rapidly recovering patient in an ICU or perhaps during the gradual recovery process of the PMV patient, as described above. 


\section{Assessing the Need for an Artificial Airway}

Once a patient has been deemed to no longer need mechanical ventilatory support (or perhaps is deemed a candidate for noninvasive ventilation), ${ }^{42,54}$ attention then turns to the assessment of the need for the artificial airway. Extubation failure can occur for reasons distinct from those that cause discontinuation failure. Examples include upper-airway obstruction or the inability to protect the airway and to clear secretions.

The risk of post-extubation upper-airway obstruction increases with the duration of mechanical ventilation, female sex, trauma, and repeated or traumatic intubation., ${ }^{3,55}$ The detection of an air leak during mechanical ventilation when the endotracheal tube balloon is deflated can be used to assess the patency of the upper airway (cuff leak test). ${ }^{55,56}$ In a study of medical patients, a cuff leak of $<110 \mathrm{~mL}$ (ie, average of 3 values on 6 consecutive breaths) measured during continuous mandatory ventilation within $24 \mathrm{~h}$ of extubation identified patients at high risk for post-extubation stridor. ${ }^{57}$ Although others have not confirmed the utility of the cuff leak test for predicting post-extubation stridor, ${ }^{58}$ many patients who develop this can be treated with steroids and/or epinephrine (and possibly with noninvasive ventilation and/or heliox) and do not necessarily need to be reintubated. Steroids and/or epinephrine also could be used $24 \mathrm{~h}$ prior to extubation in patients with low cuff leak values or who are otherwise considered at high risk. ${ }^{59}$ It is also important to note that a low value for cuff leak may actually be due to encrusted secretions around the tube rather than to a narrowed upper airway.

The capacity to protect the airway and to expel secretions with an effective cough would seem to be vital for extubation success, although specific data supporting this concept are few. Airway assessments generally include noting the quality of cough with airway suctioning, the absence of "excessive" secretions, or the frequency of airway suctioning (eg, every $2 \mathrm{~h}$ or more).,360,61 One approach uses an "airway care score," which semi-quantitatively assesses cough, gag, suctioning frequency, and sputum quantity, viscosity, and character, that predicts extubation outcomes. ${ }^{4}$ Peak cough flows of $>160 \mathrm{~L} / \mathrm{min}$ predict successful translaryngeal extubation or tracheostomy tube decannulation in neuromuscular or spinal cordinjured patients. ${ }^{62}$ Cough velocities of $0.5-1.0 \mathrm{~L} / \mathrm{s}$ have also been shown in other studies to be compatible with successful extubation. ${ }^{63}$

The importance of intact cognitive function on extubation success is controversial. Successful extubations have been reported in a select group of brain-injured, comatose patients who were judged to be capable of protecting their airways. ${ }^{4}$ However, it is difficult to extrapolate this experience to more typical ICU patients, and many would argue that some capability of the patient to interact with the care team should be present before the removal of an artificial airway. Nevertheless, a review of the literature suggests that a Glasgow coma score above 8 is compatible with successful extubation, provided that adequate airway protection capabilities exist. ${ }^{64}$

\section{The Role of Tracheostomies}

A tracheostomy offers several putative advantages over a translaryngeal airway. ${ }^{65}$ These include better patient comfort (and thus less need for sedation), ability to eat, and less infection risk. However, a tracheostomy is an invasive procedure with complications of long-term tracheal injury. In the patient who is not a simple ventilator withdrawal candidate (see above), the timing of tracheostomy placement is controversial. Proponents of "early" tracheostomy argue that in a patient judged likely to need an artificial airway for more than 21 days, the benefits of early tracheostomy outweigh any risks and justify the costs. These clinicians would thus place the tracheostomy as soon as this judgment is made. Proponents of "late" tracheostomy argue that it is difficult to predict who will need a ventilator for more than 21 days until the patient is close to the 21 day mark. Waiting until then avoids unnecessary tracheostomies.

One of the most supportive studies for "early" tracheostomies randomized 120 patients who were judged likely to need ventilator support for at least 16 days to either get an immediate or delayed tracheostomy (ie, at day 1618). ${ }^{66}$ Interestingly, the clinicians were quite accurate in predicting this need, as $83 \%$ of the patients randomized to late tracheostomy actually got one. The important outcomes showed a significant mortality benefit, pneumonia benefit, and ventilator stay benefit to early tracheostomy ( $32 \%$ vs $62 \%, 5 \%$ vs $25 \%$, and $7.6 \mathrm{~d}$ vs $17.4 \mathrm{~d}$ for early vs late, respectively).

A recent Cochrane systematic review analyzed this controversy and included 5 randomized trials (including the one above). ${ }^{67,68}$ Their conclusion was that, despite considerable methodologic heterogeneity, an early tracheostomy strategy was associated with trends in favor of early tracheostomy in mortality (49\% vs 64\%) and pneumonia (12\% vs $22 \%$ ), as well as a significant reduction in time on ventilator in favor of early tracheostomy ( $7 \mathrm{~d}$ vs $17 \mathrm{~d}$ ).

\section{Summary}

The ventilator discontinuation process is an essential component of overall ventilator management. Continued ventilator dependence can be a result of persistent illness or can be a result of poor management, and it is obviously important for the clinician to be able to assess both of these. An evidence-based task force has developed $12 \mathrm{ev}$ idence-based guidelines to help with these processes. Crit- 
ical among these is the recommendation for regular SBTs. More recent developments have focused on the importance of linking sedation reduction protocols to ventilator discontinuation protocols. Patients with repeated SBT failures are often considered to require PMV. These patients often receive tracheostomies and are probably better managed with more gradual reductions in support and gradually lengthening spontaneous breathing periods. The evidence base is growing, but the earlier guidelines are standing the test of time. Moreover, practice patterns are evolving in accordance with them. Nevertheless there is still room for improvement and still the need for further clinical studies.

\section{REFERENCES}

1. MacIntyre NR, Cook DJ, Ely EW Jr, Epstein SK, Fink JB, Heffner JE, et al. Evidence-based guidelines for weaning and discontinuing ventilator support: a collective task force facilitated by the American College of Chest Physicians, the American Association for Respiratory Care, and the American College of Critical Care Medicine. Chest 2001;120(6): 375S-395S. Also in: Respir Care 2002;47(1):29-30.

2. Ely EW, Baker AM, Dunagan DP, Burke HL, Smith AC, Kelly PT, et al. Effect on the duration of mechanical ventilation of identifying patients capable of breathing spontaneously. N Engl J Med 1996; 335(25):1864-1869.

3. Fagon JY, Chastre J, Hance AJ, Montravers P, Novara A, Gibert C. Nosocomial pneumonia in ventilated patients: a cohort study evaluating attributable mortality and hospital stay. Am J Med 1993;94(3):281-288.

4. Coplin WM, Pierson DJ, Cooley KD, Newell DW, Rubenfeld GD. Implications of extubation delay in brain injured patients meeting standard weaning criteria, Am J Respir Crit Care Med 2000;161(5): 1530-1536.

5. Tobin MJ, Guenther SM, Perez W, Lodato RF, Mador MJ, Allen SJ, Dantzker DR. Konno-Mead analysis of rib cage-abdominal motion during successful and unsuccessful trials of weaning from mechanical ventilation. Am Rev Respir Dis 1987;135(6):1320-1328.

6. Jubran A, Tobin MJ, Pathophysiological basis of acute respiratory distress in patients who fail a trial of weaning from mechanical ventilation. Am J Respir Crit Care Med 1997;155(3):906-915.

7. Frutos-Vivar F, Esteban A, Apezteguía C, Gonzalez M, Arabi Y, Restrepo MI, et al. Outcome of reintubated patients after scheduled extubation. J Crit Care 2011;26(5):502-509.

8. Epstein SK, Ciubotaru RL, Wong JB. Effect of failed extubation on the outcome of mechanical ventilation. Chest 1997;112(1):186-192.

9. Esteban A, Alía I, Gordo F, Fernández R, Solsona JF, Vallverdú I, et al. Extubation outcome after spontaneous breathing trials with T-tube or pressure support ventilation: the Spanish Lung Failure Collaborative Group Am J Respir Crit Care Med 1997;156(Pt 1):459-465.

10. Vallverdú I, Calaf N, Subirana M, Net A, Benito S, Mancebo J. Clinical characteristics, respiratory functional parameters, and outcome of a two-hour T-piece trial in patients weaning from mechanical ventilation. Am J Respir Crit Care Med 1998;158(6):1855-1862.

11. Ely EW, Baker AM, Evans GW, Haponik EF. The prognostic significance of passing a daily screen of breathing spontaneously. Int Care Med 1999;25(6):581-587.

12. Epstein SK, Ciubotaru RL. Independent effects of etiology of failure and time to reintubation on outcome for patients failing extubation. Am J Respir Crit Care Med 1998;158(2):489-493.

13. Esteban A, Frutos F, Tobin MJ, Alía I, Solsona JF, Valverdú I, et al. A comparison of four methods of weaning patients from mechanical ventilation: the Spanish Lung Failure Collaborative Group. N Engl J Med 1995;332(6):345-350.
14. Brochard L, Rauss A, Benito S, Conti G, Mancebo J, Rekik N, et al. Comparison of three methods of gradual withdrawal from ventilatory support during weaning from mechanical ventilation. Am J Respir Crit Care Med 1994;150(4):896-903.

15. Dojat M, Harf A, Touchard D, Laforest M, Lemaire F, Brochard L. Evaluation of a knowledge-based system providing ventilatory management and decision for extubation. Am J Respir Crit Care Med 1996;153(3):997-1004.

16. Hess DR, MacIntyre NR. Ventilator discontinuation: why are we still weaning? Am J Respir Crit Care Med 2011;184(4):392-394.

17. MacIntyre NR. Respiratory factors in weaning from mechanical ventilatory support. Respir Care 1995;40(3):244-248.

18. Tremblay LN, Slutsky AS. Ventilator-induced lung injury: from the bench to the bedside. Inten Care Med 2006;32(1):24-33.

19. MacIntyre NR. Patient-ventilator interactions: optimizing conventional ventilation modes. Respir Care 2011;56(1):73-84

20. Ibrahim EH, Kollef MH. Using protocols to improve the outcomes of mechanically ventilated patients. Focus on weaning and sedation. Crit Care Clin 2001;17(4):989-1001.

21. Esteban A, Anzueto A, Frutos F, Alía I, Brochard L, Stewart TE, et al; Mechanical Ventilation International Study Group et al. Mechanical Ventilation International Study Group. Characteristics and outcomes in adult patients receiving mechanical ventilation: 128 -day international study. JAMA 2002;287(3):345-355.

22. Boles JM, Bion J, Connors A, Herridge M, Marsh B, Melot C, et al Weaning from mechanical ventilation. Eur Respir J 2007;29(5): 10331056.

23. Panuelas O, Frutos-Vivar F, Fernandez C, Anzueto A, Epstein SK, Apezteguía C, et al; Ventila Group. Characteristics and outcomes of ventilated patients according to time to liberation from mechanical ventilation. Am J Respir Crit Care Med 2011;184(4):430-437.

24. MacIntyre NR, Epstein SK, Carson S, Scheinhorn D, Christopher K, Muldoon S. Management of patients requiring prolonged mechanical ventilation. Chest 2005;128(6):3937-3954.

25. MacIntyre NR. Chronic critical illness: the growing challenge to health care. Respir Care 2012;57(6):1021-1027.

26. White AC. Long term mechanical ventilation: management strategies. Respir Care 2012;57(6):889-899.

27. Yang KL. Reproducibility of weaning parameters: a need for standardization. Chest 1992;102(6):1829-1832.

28. Cook D, Meade M, Guyatt G, Griffith L, Booker L. Evidence report on criteria for weaning from mechanical ventilation. Rockville, MD: Agency for Health Care Policy and Research; 1999. Contract No. 290-97-0017.

29. Tejerina E, Esteban A, Fernández-Segoviano P, María RodríguezBarbero J, Gordo F, Frutos-Vivar F, et al. Clinical diagnoses and autopsy findings: discrepancies in critically ill patients. Crit Care Med 2012;40(3):842-846.

30. Kollef MH, Shapiro SD, Silver P, St John RE, Prentice D, Sauer S, et al. A randomized, controlled trial of protocol-directed versus physician-directed weaning from mechanical ventilation. Crit Care Med 1997;25(4):567-574.

31. Esteban A, Alía I, Tobin MJ, Gil A, Gordo F, Vallverdú I, et al. Effect of spontaneous breathing trial duration on outcome of attempts to discontinue mechanical ventilation: the Spanish Lung Failure Collaborative Group. Am J Respir Crit Care Med 1999;159(2):512-518.

32. deHaven CB, Kirton OC, Morgan JP, Hart AM, Shatz DV, Civetta JM. Breathing measurements reduces false negative classification of atachypneic pre-extubation trial failures. Crit Care Med 1996;24(6): 976-980.

33. Tanios MA, Nevins MI, Hendra KP, Cardinal P, Allan JE, Naumova EN, Epstein SK. A randomized controlled trial of the role of weaning predictors in clinical decision making. Crit Care Med 2006;34(10): 2530-2535. 
34. Yang KL, Tobin MJ. A prospective study of indexes predicting the outcome of trials of weaning from mechanical ventilation. N Engl J Med 1991;324(21):1445-1450.

35. Cohen C, Zagelbaum G, Gross D, Roussos C, Macklem PT. Clinical manifestations of inspiratory muscle fatigue. Am J Med 1982;73(3): 308-316.

36. Liu L, Liu H, Yang Y, Huang Y, Liu S, Beck J, et al. Neuroventilatory efficiency and extubation readiness in critically ill patients. Crit Care 2012;16(4):R143.

37. Jones DP, Byrne P, Morgan C, Fraser I, Hyland R. Positive endexpiratory pressure vs T-piece: extubation after mechanical ventilation Chest 1991;100(6):1655-1659.

38. Bailey CR, Jones RM, Kelleher AA. The role of continuous positive airway pressure during weaning from mechanical ventilation in cardiac surgical patients. Anaesthesia 1995;50(8):677-681.

39. Petrof BJ, Legare M, Goldberg P, Milic-Emili J, Gottfried SB. . Continuous positive airway pressure reduces work of breathing and dyspnea during weaning from mechanical ventilation in severe chronic obstructive pulmonary disease. Am Rev Respir Dis 1990;141(2):281-289.

40. Tobin MJ. Extubation and the myth of "minimal ventilator settings". Am J Respir Crit Care Med 2012;185(4):349-350.

41. Jubran A, Tobin MJ. Passive mechanics of lung and chest wall in patients who failed or succeeded in trials of weaning. Am J Respir Crit Care Med 1997;155(3):916-921.

42. Tobin MJ. Laghi F. Brochard L. Role of the respiratory muscles in acute respiratory failure of COPD: lessons from weaning failure. J Appl Physiol 2009;107(3):962-970.

43. Jubran A, Parthasarathy S. Hypercapneic respiratory failure during weaning: neuromuscular capacity vs muscle loads. Respir Care Clin N Am 2000;6(3):385-406.

44. Capdevila X, Perrigault PF, Ramonatxo M, Roustan JP, Peray P, d'Athis F, Prefaut C. Changes in breathing pattern and respiratory muscle performance parameters during difficult weaning. Crit Care Med 1998;26(1):79-87.

45. Laghi F, D'Alfonso N, Tobin MJ. Pattern of recovery from diaphragmatic fatigue over 24 hours. J Appl Physiol 1995;79(2):539-546.

46. Blackwood B, Alderdice F, Burns K, Cardwell C, Lavery G, O'Halloran P. Use of weaning protocols for reducing duration of mechanical ventilation in critically ill adult patients: Cochrane systematic review and meta-analysis. BMJ 2011;342:c7237. DOI: 10.1136/bmj.c7237.

47. Haas CF, Loik PS. Ventilator discontinuation protocols. Respir Care 2012;57(10):1649-1662.

48. Lellouche F, Mancebo J, Jolliet P, Roeseler J, Schortgen F, Dojat M, et al. A multicenter randomized trial of computer-driven protocolized weaning from mechanical ventilation. Am J Respir Crit Care Med 2006;174(8):894-900.

49. Rose L, Preseneill JJ, Johnston L, Cade JF. A randomized, controlled trial of conventional versus automated weaning from mechanical ventilation using smartcare/ps. Intensive Care Med 2008;34(10):1788-1795.

50. Girard TD, Kress JP, Fuchs BD, Thomason JW, Schweickert WD, Pun BT, et al. Efficacy and safety of a paired sedation and ventilator weaning protocol for mechanically ventilated patient in intensive care (Awakening and Breathing Controlled Trial): a randomized controlled trial. Lancet 2008;371(9607):126-134.
51. Mehta S, Burry L, Cook D, Fergusson D, Steinberg M, Granton J, et al. Daily sedation interruption in mechanically ventilated critically ill patients cared for with a sedation protocol: a randomized controlled trial. JAMA 2012;308(19):1985-92.

52. Esteban A, Ferguson ND, Meade MO, Frutos-Vivar F, Apezteguía C, Brochard L, et al; VENTILA Group. Evolution of mechanical ventilation in response to clinical research. Am J Respir Crit Care Med 2008;177(2):170-177.

53. Jaber S, Delay JM, Matecki S, Sebbane M, Eledjam JJ, Brochard L. Volume-guaranteed pressure-support ventilation facing acute changes in ventilatory demand. Inten Care Med 2005;31(9):1181-1188.

54. Hess DR. Noninvasive ventilation for acute respiratory failure. Respir Care 2013;58(6):950-969; discussion 969-972.

55. Jaber S, Chanques G, Matecki S, Ramonatxo M, Vergne C, Souche $\mathrm{B}$, et al. Post-extubation stridor in intensive care unit patients: risk factors evaluation and importance of the cuff-leak test. Intensive Care Medicine 2003;29(1):69-74.

56. Fisher MM, Raper RF. The "cuff leak" test for extubation. Anaesthesia 1992;47(1):10-12.

57. Miller R, Cole R. Association between reduced cuff leak volume and postextubation stridor. Chest 1996;110(4):1035-1040.

58. Engoren M. Evaluation of the cuff leak test in cardiac surgery patients. Chest 1999;116(4):1029-1031.

59. Jaber S, Jung B, Chanques G, Bonnet F, Marret E. Effects of steroids on reintubation and post-extubation stridor in adults: meta-analysis of randomised controlled trials. Crit Care 2009;13(2):R49.

60. Hilberman M, Dietrich HP, Martz K, Osborn JJ. An analysis of potential physiological predictors of respiratory adequacy following cardiac surgery. Thorac Cardiovasc Surg 1976;71(5):711-720.

61. Bach J, Saporto L. Criteria for extubation and tracheostomy tube removal for patients with ventilatory failure. Chest 1996;110(6): 1566-1571.

62. Chan LY, Jones AY, Chung RC, Hung KN. Peak flow rate during induced cough: a predictor of successful decannulation of a tracheotomy tube in neurosurgical patients. Amer J Crit Care 2010;19(3):278-284.

63. Su WL, Chen YH, Chen CW, Yang SH, Su CL, Perng WC, et al. Involuntary cough strength and extubation outcomes for patients in an ICU. Chest. 2010;137(4)777-782.

64. King CS, Moores LK, Epstein SK. Should patients be able to follow commands prior to extubation? Respir Care 2010;55(1):56-65.

65. Bittner EA, Schmidt UH. The ventilator liberation process: update on technique, timing, and termination of tracheostomy. Respir Care 2012;57(10):1626-1634.

66. Rumbak MJ, Newton M, Truncale T, Schwartz SW, Adams JW, Hazard PB. A prospective, randomized, study comparing early percutaneous dilational tracheotomy to prolonged translaryngeal intubation (delayed tracheotomy) in critically ill medical patients. Crit Care Med 2004;32(8):1689-1694.

67. Gomes Silva BN, Andriolo RB, Saconato H, Atallah AN, Valente O. Early versus late tracheostomy for critically ill patients. Cochrane Database Syst Rev 2012;(3):CD007271.

68. Griffiths J, Barber VS, Morgan L, Young JD. Systematic review and meta-analysis of studies of the timing of tracheostomy in adult patients undergoing artificial ventilation. BMJ 2005; 330(7502): 1243 .

\section{Discussion}

Branson: Neil, what do you think about Martin's [Tobin] recent editorial $^{1}$ that there is no such thing as minimal ventilator settings and we all ought to be doing SBTs with absolutely no support?

MacIntyre: Martin makes the case that CPAP or PSV of $5 \mathrm{~cm} \mathrm{H}_{2} \mathrm{O}$ or PAV or whatever you want, does not mimic what the patient will face withthe tube out. I think that's probably true. Therefore, in the borderline case, I think his idea to go to a T-piece is probably a good idea. Having said that, the evidence from Esteban's large 
trials ${ }^{2,3} 10$ years ago suggest that in most patients that probably isn't necessary. In our institution, for instance, we have most patients on pressure targeted ventilation, and our SBT merely involves turning the rate as low as we can (1 or 0$)$ and the pressure assist level to $5 \mathrm{~cm} \mathrm{H}_{2} \mathrm{O}$. It's easy for the therapist to do: a couple of taps on the touch-screen and you're in the SBT. But Martin is right that there are patients where you're on the fence. In those patients, especially patients who need a lot of PEEP, it might be worthwhile to do it as an unassisted T-piece SBT.

Hess: I would argue that your therapists could just as easily go to no support by setting a pressure support of zero and a PEEP of zero.

MacIntyre: Well, if you put them on zero and zero, they still have a demand valve they have to work with.

Hess: Modern ventilators don't have demand valves.

MacIntyre: They have solenoid valves. You have electronics that have to be activated. Am I right, Mark?

Rogers: ${ }^{*}$ Yes, that's correct.

Kacmarek: And the data show that the amount of effort associated with activating a solenoid is virtually nothing. If you can't tolerate activating a solenoid, then you shouldn't be liberated.

Gajic: That is true, so, yes, the pressure support and PEEP of $5 \mathrm{~cm} \mathrm{H}_{2} \mathrm{O}$ is not what you would get after extubation. Actually, the airway will get even more narrow than with the tube in. And if you have a heart failure or fluid overload, the patient could fail. Knowing from doing other work, a

\footnotetext{
* Mark Q Rogers RRT, CareFusion, Yorba Linda, California.
}

T-piece trial to find out that they would fail without some positive pressure is a waste of time: you just extubate them to NIV according to NAVA [neurally adjusted ventilatory assist] criteria. In a patient with ischemic cardiomyopathy, instead of wasting your time on an SBT with a T-piece, if they can cough, you extubate them to NIV and then wean that slowly a few hours at a time until it's just at night, and then in a few days, it's gone.

MacIntyre: I think Dean reviewed ${ }^{4}$ this very nicely, and, Ognjen, I agree with you that if they're intubated for heart failure reasons or COPD, that's probably a legitimate thing to do. However, in a patient with other forms of respiratory failure, I would be concerned about extubating them to NIV if they haven't made me comfortable with an SBT.

Schmidt: My question is about the duration of the SBT. If I remember correctly, there was no difference between 30 minutes and 120 minutes. The study was done in acute patients, and I think you quite correctly said it's a totally different population when you go to more than 21 days. But in patients who have been ventilated for 7 to 14 days, I don't think 30 minutes is enough to predict extubation success. I don't know if anything in the literature supports the 2-hour SBT I perform. And I don't know whether I'm right, or when Bob says to do 90 minutes, he might be right. What's your guidance?

MacIntyre: That's why the recommendation from the task force is 30120 minutes. Some patients are clearly ready to go, and at 30 minutes you know it.

Schmidt: Or 5 minutes if you're in the operating room.

MacIntyre: Yes, but I' $m$ in the ICU, and I like to see it for about $30 \mathrm{~min}$ utes before I'm really comfortable it's going to work. Many times you're not really sure at 30 minutes. They're a little more tachycardic, they look a little uncomfortable, and their oxygen saturation drops a smidge: then you want to keep the SBT going. I think the 120-minute mark is a good end point, because if they're still having difficulties after 2 hours and they're not comfortable, then I'm not comfortable. They go back on the ventilator and we try again tomorrow.

Kallet: I think there are other issues that need to be examined. I think the idea of putting a patient you're not going to extubate back on ventilatory support after 2 hours is kind of misguided if they're not showing signs of failure. As the point person for the SBT weaning protocol at my hospital, it's been beaten into me by everyone to relax that aspect of the protocol.

Let's take a patient who's been intubated for 2 weeks and had several bouts of sepsis. A lot of clinicians are not willing to pull the tube after the patient passes their first 2-hour SBT, even if they appear to be tolerating it, so why not let them go until the point that they declare themselves? I say that because, in the literature on disuse atrophy of the respiratory muscles, ${ }^{5}$ signs of respiratory distress develop before overt fatigue, and it's high-frequency fatigue that occurs first. If you put the patient back on support when they show signs of respiratory distress, at least the physiologic studies show that the recovery from high-frequency fatigue is very rapid, in a matter of hours.

So if you don't push them past that point, chances are you won't appreciably overtax the respiratory muscles and set back their progress. The idea of resting the patient is very prudent, but the pushback that I've gotten politically from faculty on the SBT protocol mandating that the patient goes back to full ventilatory support regardless after 2 hours - they just won't go with that. 
MacIntyre: Rich, I tried to bring it out, but maybe I didn't do as well as I thought. This 30-120 minute time frame has been studied in the acute ICU setting. It has not been studied in the PMV group (more than 21 days) or the gray zone that Uli mentioned (12 to 20 days). In the PMV patients, all the authorities (there are no RCTs) would argue that "weaning" down to half of the original support level should be done before reinstituting SBTs. However, under these conditions the patient is usually tracheotomized, and SBT takes on a different meaning, with different rules. And you're exactly right: you push them until they declare themselves. That makes perfect sense in the PMV patients.

Kacmarek: Neil, let me push back on you about the automated weaning approach. Do $100 \%$ of your patients who need an SBT every day get it?

MacIntyre: We have a scorecard system.

Kacmarek: We do too.

MacIntyre: It's not a very good one, which is why I hope the manufacturers can help me out on this. It's a snapshot. One of our assistant directors goes around unannounced, and does spot checks, so to speak. They pick an arbitrary ICU and just look at that day or over the course of a week. And our batting average is 80 to $90 \%$. They tell me that's terrific. We're still missing 10 to $20 \%$, but I think 80 to $90 \%$ is not bad.

Kacmarek: But you're in a top-level institution, and I would expect that from you. What percentage of community hospitals come anywhere near 80 or $90 \%$ ? My point is that the Lellouche study, ${ }^{6}$ which showed that $50 \%$ of the SBTs in the control group were not performed correctly, is the clinical reality. That's what occurs in the vast majority of ICUs every day. So if an approach like SmartCare works as well as that or better, then it should be utilized. We should not throw it out the window because all it's doing is what we don't have the time, or are too distracted, or forget to do. It can't do anything but make care better for our patients.

MacIntyre: I partially agree with you. The beauty of SmartCare is that it reminds us it's time for an SBT. My concern with SmartCare is that it requires a certain reduction of support before it will give you the signal.

Kacmarek: But you can intervene and do your SBT before it gets to that point.

MacIntyre: Yeah, but if you're arguing that its advantage is that it reminds you - if it's not going to remind me until I reduce the support a certain amount - that could delay the SBT.

Kacmarek: If I remember correctly, the algorithm utilized in Europe allows you to adjust those thresholds. The algorithm not only advises you: it does the SBT and notifies you whether the patient passed or failed, and you decide whether to extubate.

MacIntyre: I think we will both agree that the SBT prompts are good, and that unnecessary delays of SBTs could be harmful. You're right: perhaps you can set it up so that's not going to be an issue, but I find it just as easy to go to the screen each day and just do the SBT.

Kacmarek: I agree. My point is, the reality in the majority of institutions is that that doesn't happen the way it should.

Marini: Neil, I agree with almost $100 \%$ of what you said. But there are patients, particularly those with cardiac disease, psychiatric problems, discoordinated breathing, et cetera, who don't give you the same response to an SBT at 6:00 AM as they do at 1:00 PM, after they've been cleaned up and are fully awake. So a second SBT during the day makes sense to me, in part because that's what we do all the time. We say, "that person should have been ready; they looked ready yesterday, but this morning they failed, so let's try it again later." I don't go back to resting them fully. I make them work a little bit, because I think a gradual reduction in support may be needed in people who have cardiac disease. Such problems surface as you go forward. Sudden discontinuation of support for them is stressful, and that abrupt challenge often produces numerically acceptable breathing patterns.

And with regard to parameters, what should we do or pay attention to? Do we measure vital capacity, inspiratory capacity? We're after reserve, aren't we? Suppose the minute ventilation is $12 \mathrm{~L} / \mathrm{min}$ right now, but during sleep a few hours before it was $6 \mathrm{~L} / \mathrm{min}$. Doesn't that encourage you to think there's some physiologic reserve to call upon? I'm raising these questions because that's the way I practice, but maybe I'm wrong.

MacIntyre: No, you're not wrong, John. The large analysis of the Esteban survey ${ }^{7}$ suggested, I think it, that two thirds of patients were so-called "simple" weans.

\section{Kacmarek: It was $75 \%$.}

MacIntyre: Right. I'm talking about that population. And then another 20\% or so take 2-7 days of weaning. My principles I talked about probably apply to them as well. But you're talking about the $6 \%$ who become more problematic, and they do need to be treated individually. They've got issues that may be much more complex, and trying to force them into rigid boxes is probably inappropriate. I think there are numbers we can follow. The weaning parameters the McMaster report $^{8}$ showed us, I think, are good guidelines, but they're just guidelines. 
I get concerned if we make them absolute thresholds that state: below this you can go, and above this you cannot go.

Marini: Me too, partly because often we're dealing with patients who have poor respiratory-system compliance. They start from a rapid-shallowbreathing index [breathing frequency divided by tidal volume] of 80 with pressure support, when they're perfectly comfortable. As they exercise during the SBT, they may comfortably go to 120. I've extubated patients with rapid-shallow-breathing indexes greater than 150 !

MacIntyre: I agree. The Tanios ${ }^{9}$ study clearly showed that using that number as an absolute threshold produced longer stay on the ventilator.

Turner: What do you think our target should be for the extubation failure rate, to say we're doing a good job?

MacIntyre: Extubation failure rate is a nice monitoring tool for how your unit is performing. Should the reintubation rate be zero? Well, if it's zero, chances are you're waiting too long and missing a good number of patients. Should it be 50\%? Well, that seems pretty high, if it's just a flip of a coin. A couple years ago Scott Epstein did a large survey ${ }^{10}$ of ICUs across North America, and his data suggest that the standard today is somewhere in the 10 to $15 \%$ range. I think that's probably a reasonable rate.

Schmidt: I'm not so sure. I was pretty sure, but now looking through the recent literature on problems with reintubation, the mortality associated with reintubation, and so forth. I'm not saying zero, but I'm not so sure when you're at $15 \%$, which means $15 \%$ of our patients we give a 5 -times higher mortality risk. Maybe they had that before, but I think we have to be careful to say that an SBT means ex- tubation automatically. Why can't we let them sit for a day until they're ready to be extubated?

MacIntyre: They're two entirely different assessments: one is whether they need the ventilator, and the other is whether they need the tube. All too often those two assessments get lumped together.

Schmidt: Exactly: I agree. This is one of our biggest problems and challenges. To really be ready to extubate, maybe my anesthesiology colleagues are better than I am, because we're still struggling.

Kacmarek: The thing that always concerns me about those numbers is that they're lumped together based on RCTs that were primarily done in medical patients. There's some surgical patients in there, but every one of the studies had a large number of medical patients. And you'd expect the medical ICU and cardiac surgical ICU reintubation rates to be dramatically different, as you would for the pediatric ICU. I expect them to be higher in a medical unit than in a surgical unit. I don't think you can look across the board and say that $10 \%$ or $15 \%$ is appropriate. It has to be for your unit and your trends over time. Obviously, we all should be working toward the lowest reasonable reintubation rate and looking at the duration of mechanical ventilation.

MacIntyre: This is one of our quality measures back home. For several years now at Duke, both our medical and surgical ICUs have consistently been in the $10-12 \%$ range.

Kacmarek: Your cardiac surgical patients?

MacIntyre: No, our general surgical. We have not measured this in the cardiothoracic unit. I agree with you that in the cardiothoracic unit it's probably much lower.

Kacmarek: My point is that I think you've got to do it unit by unit. Not use an institutional number.

Branson: Bob, how many and which of the ICUs at Massachusetts General Hospital are using SmartCare?

Kacmarek: None. Because, like Neil, we think that we know what we're doing and that we can manage patients appropriately without SmartCare. But, like Neil, we are not your typical community hospital.

Gajic: We should distinguish the need for airway protection from the need for positive pressure, which can be applied noninvasively. If the patient can cough, he or she can be extubated to NIV. If the patient can't cough, or fails extubation to NIV, then tracheostomy may be the best option. This may be a sound approach to get to a less than $10 \%$ extubation failure rate, even in medical patients, and would probably prevent some of the noise in other things that we do.

Kallet: I want to follow up something John said, that I think touches on the issue of clinician automatic pilot. You do an SBT; the patient fails after only a couple minutes; you put them back on full support. If you suddenly withdraw ventilatory support and the workload is abruptly transferred to the patient, it tends to freak some of them out, which is one reason I don't necessarily like the idea of pairing the SBT with the sedation interruption. If a patient does freak out on SBT, just put them on a pressure support level where they're comfortable and see over 5 or 10 minutes if you can gradually titrate the pressure support back down to where it's an SBT. If they can't adapt after about $10 \mathrm{~min}$ utes, I think you have your answer.

But I also think that when we put a protocol like SBT into operation, we 
don't anticipate some important practical ramifications. I totally sympathize with the therapist who says, "I've got 5 SBTs to do this morning, along with everything else I'm responsible for!" Under those circumstances it's understandable that when a patient initially fails an SBT, the clinician aborts it without thinking in depth as to why. When the physician later asks, "Why did they fail?" the unhelpful answer is, "Well, they didn't tolerate it!" and that's been causing some friction. So the recent iteration of the SBT protocol at our hospital allows up to 30 minutes to get the patient accommodated.

MacIntyre: One nice thing about the approach I described is that you can dial the pressure down: you don't have to go from 15 to 5. You can go from 15 to 12 to 10 in a few minutes, while you're standing there. And, John, just to clarify a point that you made, when I say that in between SBTs we keep them comfortable, it is still with a ventilator work load. The diaphragm is not designed to "rest." It's not like other skeletal muscles that can go to sleep at night and be fine the next day. Instead, the diaphragm is very much like the heart, which also is not designed to be totally rested. You don't treat heart failure with asystole: at least not for very long. I'm sure you and I agree that keeping the patient comfortable with a reasonable level of activity is important in between the SBTs.

Marini: I think a lot of areas of intensive care are going toward less ag- gressively supported care and more towards trying to adapt patients to their diseases. And part of that adaptation is episodic stress. In recreational exercise we stress ourselves much more than when at rest, and we build endurance, strength, and muscle. If we keep these people fully supported and doing only gentle amounts of work, and then ask them to do the equivalent of athletic exercise during the SBT, we may be setting them up for failure. The old idea of periodically doing sprints (SBTs) may hold water. If you do them several times each day, leading up to the final evaluation for extubation, perhaps such conditioning and training might re-educate the neural drive and improve muscular efforts. Just a thought.

MacIntyre: That's a good thought, and I've often wondered if we could exercise and train the muscle recovering from a respiratory failure. At some points I've thought maybe we could, but I haven't see much evidence to suggest that it's possible. I'm still not sure if it's possible to train or exercise a muscle that's been beaten and battered by the systemic inflammatory response syndrome and all the metabolic derangements that go along with it. But I'm keeping an open mind.

\section{REFERENCE}

1. Tobin MJ. Extubation and the myth of "minimal ventilator settings". Am J Respir Crit Care Med 2012;185(4):349-350.

2. Esteban A, Alía I, Tobin MJ, Gil A, Gordo F, Valleverdú I, et al. Effect of spontaneous breathing trial duration on outcome of attempts to discontinue mechanical ventilation. Spanish Lung Failure Collaborative
Group. Am J Respir Crit Care Med 1999; 159(2):512-518.

3. Esteban A, Alía I, Gordo F, Fernández R, Solsona JF, Valleverdú I, et al. Extubation outcome after spontaneous breathing trials with T-tube or pressure support ventilation. Spanish Lung Failure Collaborative Group. Am J Respir Crit Care Med 1997;156(2 Pt 1):459-465. Erratum in: Am J Respir Crit Care Med 1997;156(6):2028.

4. Hess DR. The role of noninvasive ventilation in the ventilator discontinuation process. Respir Care 2012;57(10):1619-1625.

5. Kallet RH. Patient-ventilator interaction during acute lung injury, and the role spontaneous breathing. Part 1: respiratory muscle function during critical illness. Respir Care 2011;56(2):181-189.

6. Lellouche F, Mancebo J, Jolliet P, Roeseler J, Schortgen F, Dojat M, et al. A multicenter randomized trial of computer-driven protocolized weaning from mechanical ventilation. Am J Respir Crit Care Med 2006; 174(8):894-900.

7. Peñuelas $\mathrm{O}$, Frutos-Vivar $\mathrm{F}$, Fernández $\mathrm{C}$, Anzueto A, Epstein SK, Apezteguía C, et al. Characteristics and outcomes of ventilated patients according to time to liberation from mechanical ventilation. Am J Respir Crit Care Med 2011;184(4):430-437.

8. MacIntyre NR, Cook DJ, Ely EW Jr, Epstein SK, Fink JB, Heffner JE, et al. Evidence-based guidelines for weaning and discontinuing ventilator support: a collective task force facilitated by the American College of Chest Physicians; the American Association for Respiratory Care; and the American College of Critical Care Medicine. Chest 2001;120(6 Suppl):375S-395S; Respir Care 2002;47(1):69-90.

9. Tanios MA, Nevins MI, Hendra KP, Cardinal P, Allan JE, Naumova EN, Epstein SK. A randomized controlled trial of the role of weaning predictors in clinical decision making. Crit Care Med 2006;34(10): 2530-2535.

10. Frutos-Vivar F, Ferguson ND, Esteban A, Epstein SK, Arabi Y, Apezteguía C, et al. Risk factors for extubation failure in patients following a successful spontaneous breathing trial. Chest 2006;130(6):16641671. 